\title{
EPR imaging of dose distributions aiming at applications in radiation therapy
}

\author{
Eva Lund, Emelie Adolfsson, Natallia Kolbun and Håkan Gustafsson
}

\section{Linköping University Post Print}

\begin{abstract}
N.B.: When citing this work, cite the original article.
\end{abstract}
Original Publication:

Eva Lund, Emelie Adolfsson, Natallia Kolbun and Håkan Gustafsson, EPR imaging of dose distributions aiming at applications in radiation therapy, 2014, Radiation Protection Dosimetry, (159), 1-4, 130-136.

http://dx.doi.org/10.1093/rpd/ncu142

Copyright: Oxford University Press (OUP): Policy B - Oxford Open Option A http://www.oxfordjournals.org/

Postprint available at: Linköping University Electronic Press

http://urn.kb.se/resolve?urn=urn:nbn:se:liu:diva-109275 


\title{
EPR imaging of dose distributions aiming at applications in radiation therapy
}

\author{
E Lund $^{1}$, E Adolfsson ${ }^{2}, \mathrm{~N}_{\text {Kolbun }}{ }^{1}$ and $\mathrm{H}_{\text {Gustafsson }}{ }^{3,4}$ \\ ${ }^{1}$ Radiation Physics, Department of Medical and Health Sciences, Linköping University, Linköping, \\ Sweden \\ ${ }^{2}$ Radiation Physics, Department of Medical and Health Sciences, Linköping University and Div. of \\ Radiation Physics, University Hospital, Linköping Sweden. \\ ${ }^{3}$ Department of Biomedical Engineering (MTÖ), County Council of Östergötland, Radiation Physics, \\ Department of Medicine and Health Sciences, Linköping University, Linköping, Sweden \\ ${ }^{4}$ Center for Medical Image Science and Visualization, CMIV, Linköping University, Linköping, \\ Sweden.
}

Corresponding author:

Eva Lund, e-mail Eva.Lund@liu.se

Tel '4613286851

\begin{abstract}
A one dimensional EPR imaging method for visualization of dose distributions in photon fields has been developed. Pressed pellets of potassium dithionate were homogenously irradiated in a ${ }^{60} \mathrm{Co}$ radiation field to $600 \mathrm{~Gy}$. The EPR analysis was performed with an $\mathrm{X}$ Band (9.6 GHz) Bruker E540 EPR and EPR imaging spectrometer equipped with an E540 GC2X two axis X band gradient coil set with gradients along y axis (along the sample tube) and $\mathrm{z}$ axis (along B0) and an ER 4108TMHS resonator. Image reconstruction, including deconvolution, base-line corrections and corrections for the resonator sensitivity was performed using an in-house developed Matlab code for the purpose to have a transparent and complete algorithm for image reconstruction. With this method it is possible to visualize a dose distribution with an accuracy of about $5 \%$ within $\pm 5 \mathrm{~mm}$ from the center of the resonator.
\end{abstract}




\section{Introduction}

Electron paramagnetic resonance, EPR, has long been used to map dose distributions in radiation therapy as for instance experimental verification of dose distributions around brachytherapy sources using alanine/agarose gel and alanine film (1) or lithium formate dosimeter tablets (2). An example of application in external beam therapy has been for verification measurements of intensity modulated radiotherapy (IMRT) dose plans, using lithium formate tablets (3). Waldeland et al. (4) placed small lithiumformate dosimeters (3 $\mathrm{mm}$ diameter) in a grid for comparison with dose plans for stereotactic radiosurgery and found fairly good correspondence between calculated and measured doses except for in the penumbra region. Imaging with MR gel dosimetry reaches a resolution of about $1 \mathrm{~mm}$ and for GafChromic films the resolution is limited by the pixel size of the read out equipment; the film resolution is extremely high due to the small average size of the crystals.

Electron paramagnetic resonance imaging (EPRI) is an attractive method for visualization of dose distributions in radiation therapy especially in steep dose gradients. EPRI has successfully been used to image dose distributions in close vicinity of brachy therapy sources $(5,6)$ and in irradiated bone (7). Anton and Selbach, (8) measured dose distributions in alanine but found because of broad line width that the deviation between measured and calculated dose distribution was too high, $10 \%$, for clinical use. EPRI has also been used to image dose and LET (linear energy transfer) distributions in potassium dithionate phantoms irradiated with heavy charged particles (9).

The spatial resolution for EPRI is depending on the line width of the EPR spectrum according to: $\mathrm{d}=\mathrm{lw} / \mathrm{grad}$, where $\mathrm{d}$ is the smallest distance for resolving two separate objects, $1 \mathrm{w}$ is the linewidth and grad the gradient strength $(10,11)$. The best resolution achievable for the dosimeter materials used in the present study without further deconvolution will thus be 0.3 $\mathrm{mm}$ for potassium dithionate with a spectral line width of $5 \mathrm{G}$ and $0.8 \mathrm{~mm}$ for lithium formate with a line width of $14 \mathrm{G}$ in a gradient field of $170 \mathrm{G} / \mathrm{cm}$. Anton and Selbach (8) achieved a much higher resolution $0.1 \mathrm{~mm}$ for alanine in a lower gradient field of $48 \mathrm{G} / \mathrm{cm}$ as the best comparison between the image reconstruction and a Monte Carlo simulated curve convolved with a point spread function.

High resolution can be achieved for narrow EPR lines, but also other factors affect the image quality. EPR imaging artifacts can occur, especially when high gradient field strengths are used to achieve high image resolution, caused by e.g. low signal to noise ratio (SNR) and 
imperfections in the resonator sensitivity profile. Simulations are recommended to increase the understanding of the different components in imaging (12) For imaging of dose distributions without prior knowledge of the size of the object it is of extreme importance to avoid imperfections in the baseline following the low SNR for the spectra in the gradient field. To test the different factors affecting the imaging homogenously irradiated pressed tablets of potassium dithionate were used taking advantage of the narrow line-width of the EPR signal and pressed tablets of lithium formate were used for comparison.

The aim of this investigation was to step by step evaluate the reconstruction of $1 D$ EPR images for the purpose of visualizing steep dose gradients in radiation therapy with a resolution well below $1 \mathrm{~mm}$.

\subsection{Principle of EPR imaging}

The microwave radiation is absorbed when the microwave energy corresponds exactly to the difference in energy between the two spin states of an unpaired electron in the magnetic field $\mathrm{B}_{0}, h v=g_{e} \mu_{B} B_{0}$ where $\mathrm{h}$ is Planck's constant, $\mathrm{g}_{\mathrm{e}}$ the electron g-factor and $\mu_{\mathrm{B}}$ the Bohr magneton. When applying a gradient field along the magnetic field, the y direction; the field at which resonance occurs is

$$
B^{\prime}(y)=B_{0}-G_{y} \cdot y
$$

where Gy is the gradient field at position y.

For a 1D image the radical density is $\rho_{\vec{G}}(\mathrm{y})$ in a plane perpendicular to the gradient field at position $y$ and the total signal from the object from $y_{1}$ to $y_{2}$ is then

$$
S_{\vec{G}}\left(B_{0}\right)=\int_{y_{1}}^{y_{2}} \rho_{\vec{G}}(y) f\left(B_{0}-G_{y} \cdot y\right) d y
$$

The profile density function is obtained by deconvolution of the total signal with the line shape function fobtained at zero gradient followed by an inverse Fourier transformation back to the spatial domain, eq 3.

$$
\begin{aligned}
& \rho_{\vec{G}}^{\prime}(y)=\operatorname{IFT}\left[F T\left(S_{\vec{G}}\right) \cdot W / F T(f)\right] \\
& \rho_{\vec{G}}^{\prime}(y)=\rho_{\vec{G}}(y) * F T(W)
\end{aligned}
$$

The signal to noise ratio is rather low for the spectrum obtained at a high gradient and to avoid division with zero a low pass filtering is applied in Fourier space, W, eq 4. We have 
chosen a Gaussian window function, $\mathrm{W}$, and the width has to be chosen as a compromise between high frequency noise reduction and the resolution.

\section{Materials and methods}

\subsection{Chemicals and Synthesis}

Polycrystalline lithium formate monohydrate $(98 \%)\left(\mathrm{HCO}_{2} \mathrm{Li} \mathrm{H}_{2} \mathrm{O}\right)$ was obtained from SigmaAldrich. Barium dithionate was synthesized according to standard methods. Potassium dithionate was synthesized using barium dithionate and prepared by mixing equivalent amounts of potassium sulphate and barium dithionate in water solutions. The solution obtained after filtering was kept at room temperature until the water had evaporated to obtain a crystalline precipitate of potassium dithionate.

\subsection{Preparation of samples}

Synthetized polycrystalline potassium dithionate and lithium formate respectively were crushed in a mortar and sieved to grain size $90 \mu \mathrm{m}<\mathrm{d}<180 \mu \mathrm{m}$ using an Endecotts MINOR test sieve shaker. Potassium dithionate and lithium formate dosimeters were prepared in the same way. A homogenous mixture of $90 \%$ (mass) of the dosimeter material and $10 \%$ paraffin were made by repeated cycles of heating to allow melting of the paraffin (the temperature was always well below the melting point of lithium formate) and mixing in room temperature. Tablets, $5 \mathrm{~mm}$ height and $4.5 \mathrm{~mm}$ diameter, were pressed using a table-top tablet press.

\subsection{Irradiation}

The irradiation to a dose of $600 \mathrm{~Gy}$ was performed at $100 \mathrm{~cm}$ distance from the ${ }^{60} \mathrm{Co}$ source with $2 \mathrm{~cm}$ build up and turned $180^{\circ}$ after half irradiation time at the irradiation facility at the Swedish Secondary Standards Dosimetry Laboratory, SSM, Stockholm.

\subsection{EPRI measurement}

1D EPRI measurements were performed with an X Band (9.6 GHz) Bruker E540 EPR and EPR imaging spectrometer equipped with an E540 GC2X two axis X band gradient coil set with gradients along y axis (along sample tube) and $\mathrm{z}$ axis (along B0) and with a resonator of type ER 4108TMHS. 
Spectrometer settings: Modulation frequency: $100 \mathrm{kHz}$, modulation amplitude: $5 \mathrm{G}$, time constant: $20.48 \mathrm{~ms}$, sweep time: $40.42 \mathrm{~s}$, sweep width $510 \mathrm{G}$, number of scans: 6, 1024 sampling points and gradient field $170 \mathrm{G} / \mathrm{cm}$

\subsubsection{Experimental performance}

Unirradiated and homogenously irradiated tablets, $600 \mathrm{~Gy}$, of potassium dithionate and litium formate $5 \mathrm{~mm}$ height, respectively, were stacked at different positions in a Wilmad sample tube Q-5M-6M-O-200 m-FB (inner diameter $5 \mathrm{~mm}$, flat bottom) up to the total length of 20 $\mathrm{mm}$, i.e. $4 * 5 \mathrm{~mm}$. Measurements were also performed on $20 \mathrm{~mm}$ of stacked unirradiated 5 $\mathrm{mm}$ tablets of the same dosimeter materials.

All combinations of tablets (for all experiments the total sample length was $20 \mathrm{~mm}$ ) were measured both without gradient and with a gradient of $170 \mathrm{G} / \mathrm{cm}$.

\subsection{Post processing}

The EPR data were imported into Matlab using the Easyspin toolbox (13). The dose profiles were calculated using an in-house developed Matlab code (version R2011a, Math Works, Inc).

For base line corrections the spectra from the unirradiated $20 \mathrm{~mm}$ rod measured at $170 \mathrm{G} / \mathrm{cm}$ and without gradient were subtracted from the corresponding spectra from irradiated samples at $170 \mathrm{G} / \mathrm{cm}$ and without gradient respectively.

The spatial information (the dose profile) was separated from the spectral information (the EPR spectrum) using deconvolution in the Fourier domain; The base line corrected spectrum obtained at $170 \mathrm{G} / \mathrm{cm}$ was low pass filtered with a Gaussian function; fwhm 75-100 of 1024 sampling points and deconvoluted with the spectrum without gradient. Finally an inverse transformation was made to return to the spatial domain, see eqs 3 and 4 .

\subsubsection{Corrections for inhomogeneous EPR resonator sensitivity profile}

It is well known that EPR resonator sensitivity profiles are only approximately constant along the $y$ axis in a very narrow region around the center of the EPR resonators (14). This also true for the EPR resonator (ER 4108TMHS) used in the experiments presented in this work. Therefore a polynomial function was developed and implemented in the Matlab code to correct for the inhomogeneous resonator sensitivity along the y axis in order to be able to 
faithfully deduce $1 \mathrm{D}$ dose profiles. The developed code used for correction of the inhomogeneous sensitivity profile was also compared to an experiment using a small crystal of DPPH in the center of a $2.5 \mathrm{~mm}$ unirradiated dosimeter tablet and this tablet was placed in different positions in the stack of unirradiated dosimeters (for establishing a sensitivity function with the same load in the resonator as during imaging) along the sample tube to determine the sensitivity from $-10 \mathrm{~mm}$ to $+10 \mathrm{~mm}$ in steps of $2.5 \mathrm{~mm}$.

\section{Results}

The raw spectra, (in a gradient strength of $170 \mathrm{G} / \mathrm{cm}$ and without gradient), from an irradiated potassium tablet $5 \mathrm{~mm}$ long and surrounded by $10 \mathrm{~mm}$ on one side and $5 \mathrm{~mm}$ on the other side of unirradiated potassium dithionate are shown in figure 1. The tablet position was just above the center of the resonator. In figure 2 the central part of the spectra is magnified to show the quite small differences in the background which turned out to have a severe impact on the baseline of the dose distributions visualized after deconvolution.

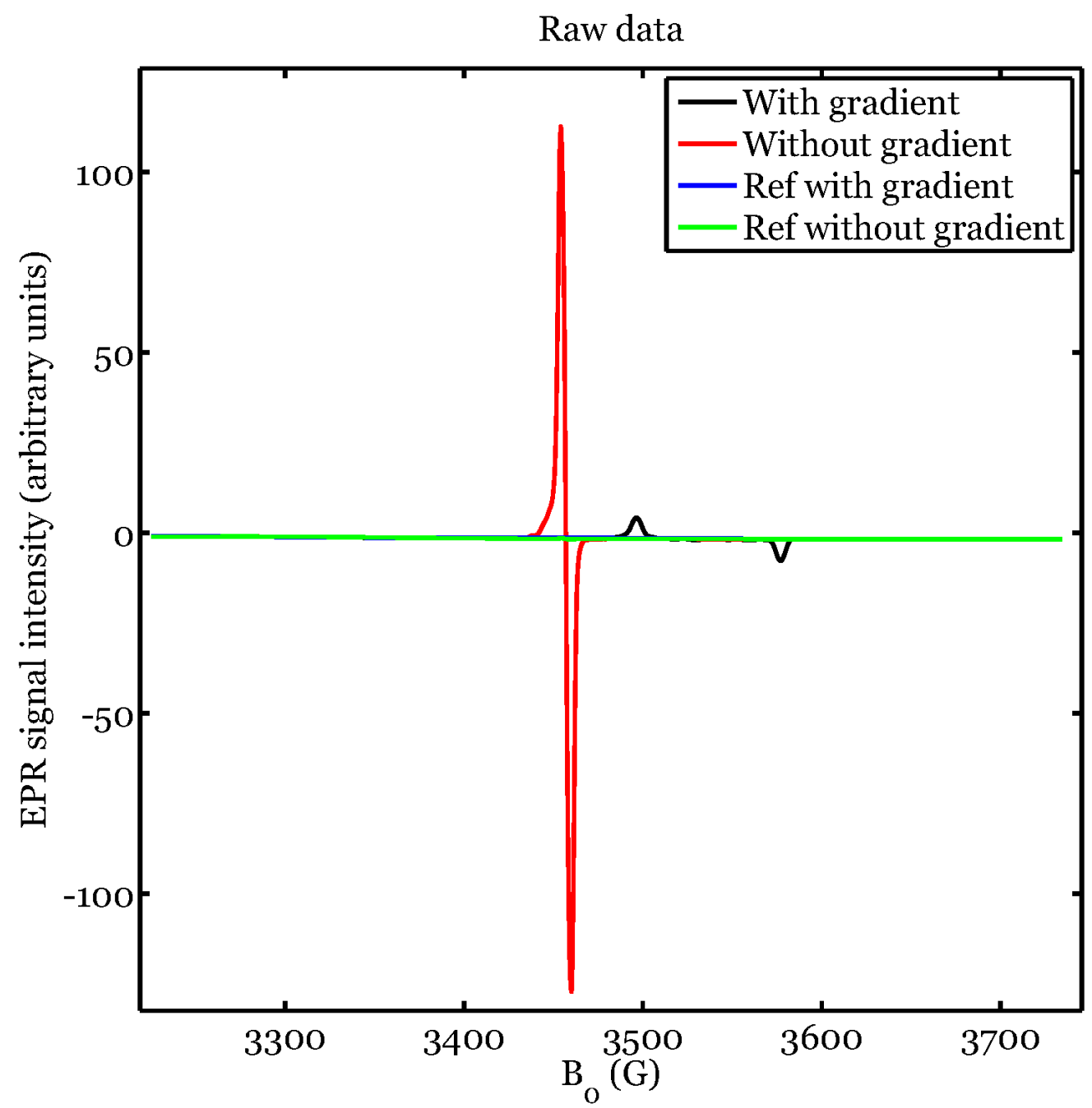




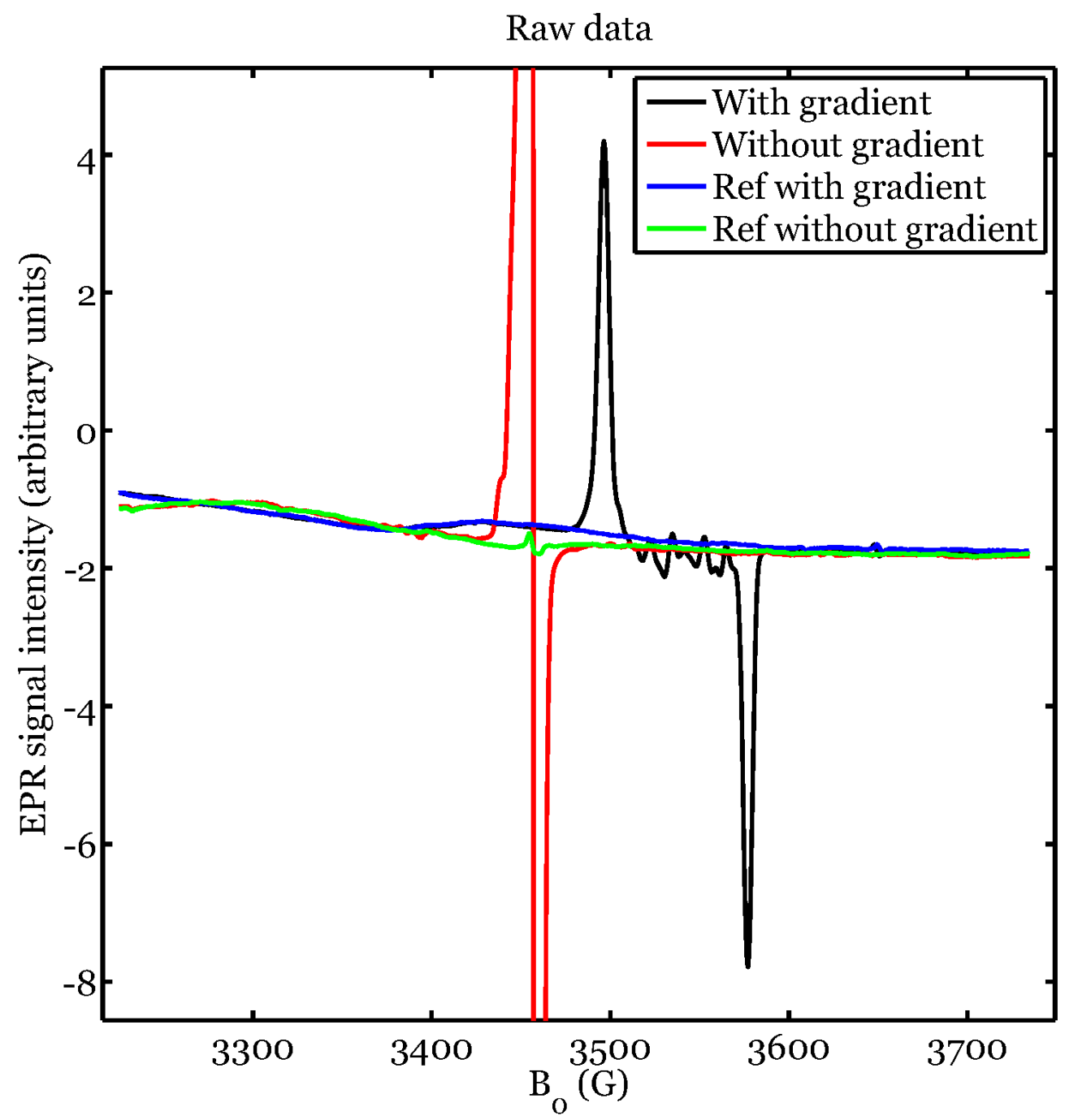

A direct deconvolution without any corrections is far from satisfactory as shown in figure 3 . Small base line shifts introduce large errors in the dose distribution after deconvolution. The baseline corrections substantially improve the vizualisation of the density profile. 
Direct deconvolution of raw data. No corrections.

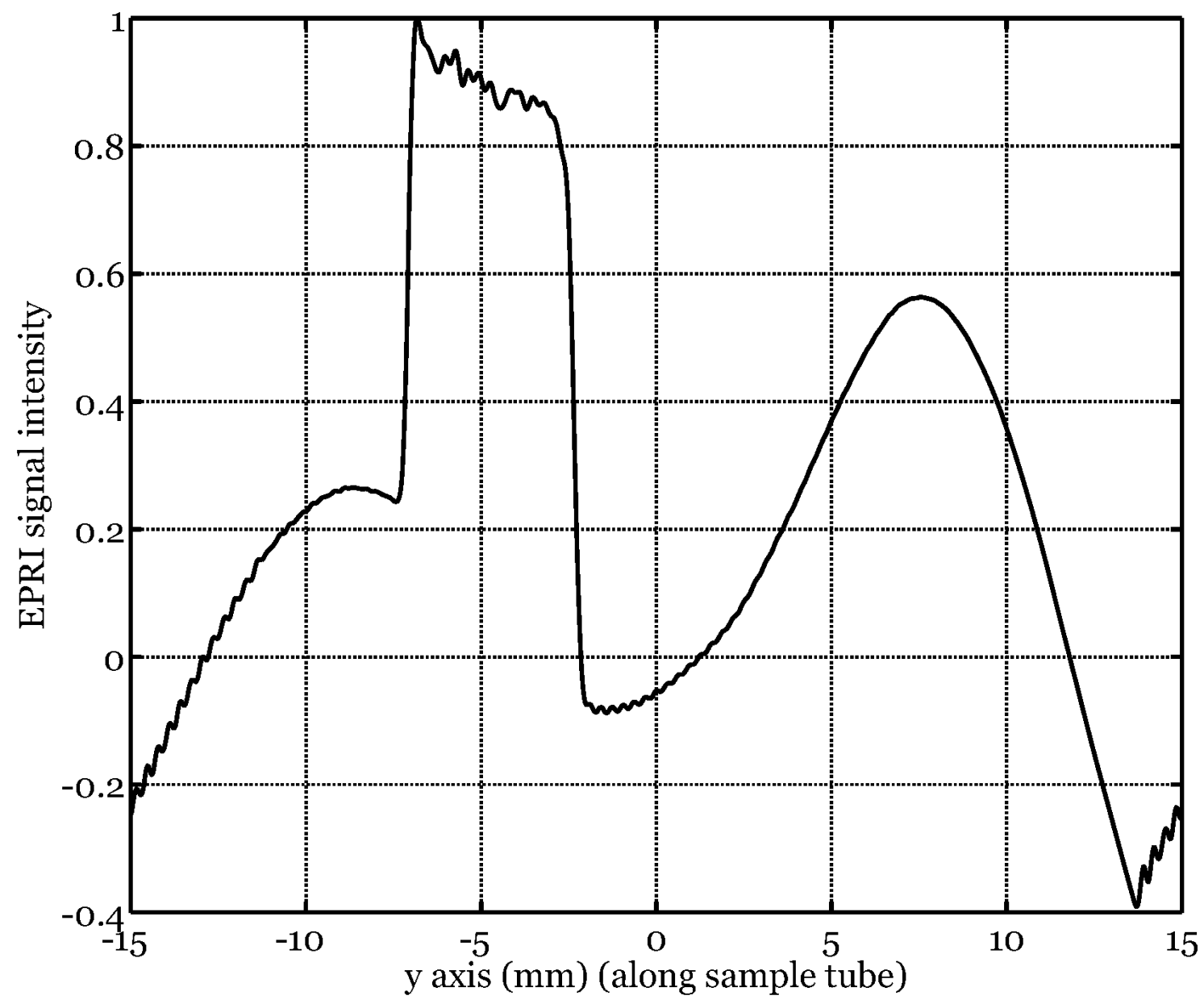

The base line corrected radical density profile together with the correction function for the inhomogenous sensitivity of the resonator is shown in figure $4 \mathrm{a}$ and the final result after this in figure $4 b$. 
After base line correction.

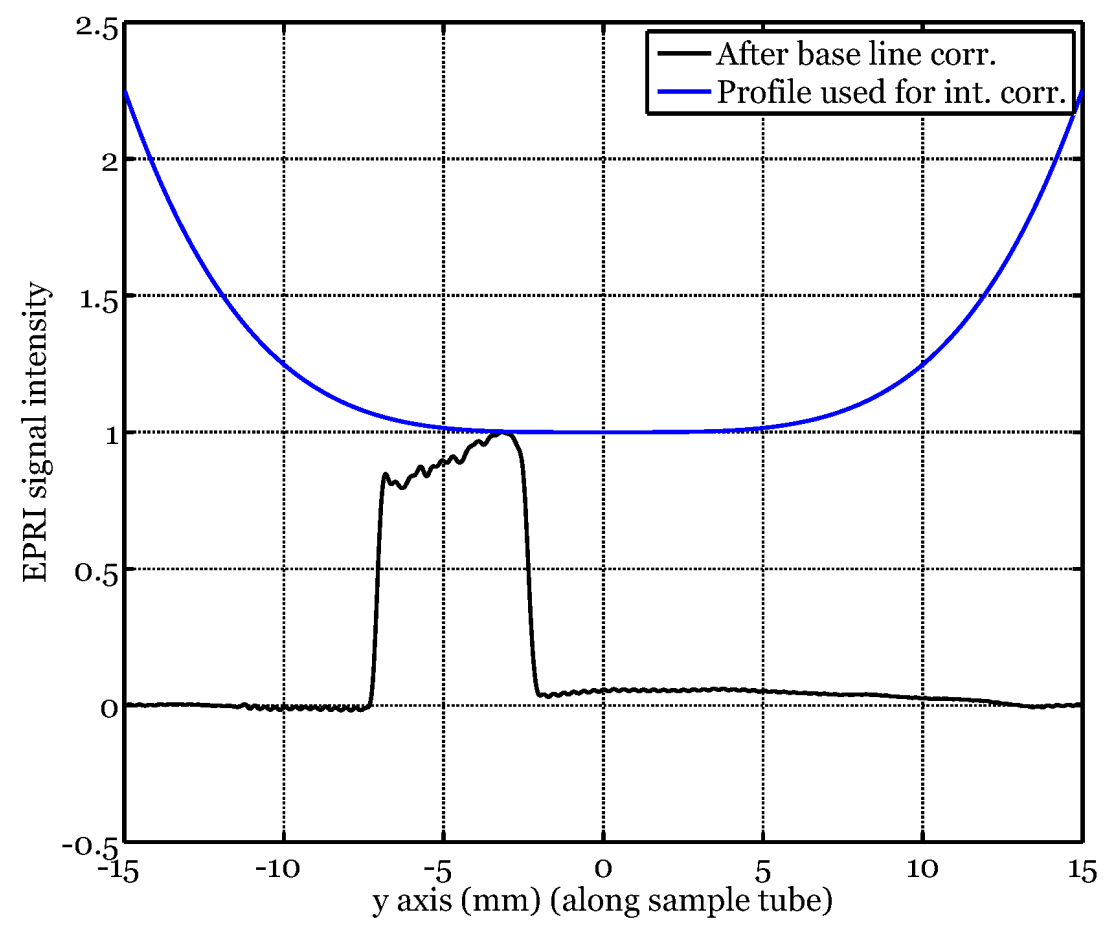

Both base line corr. and int. corr.

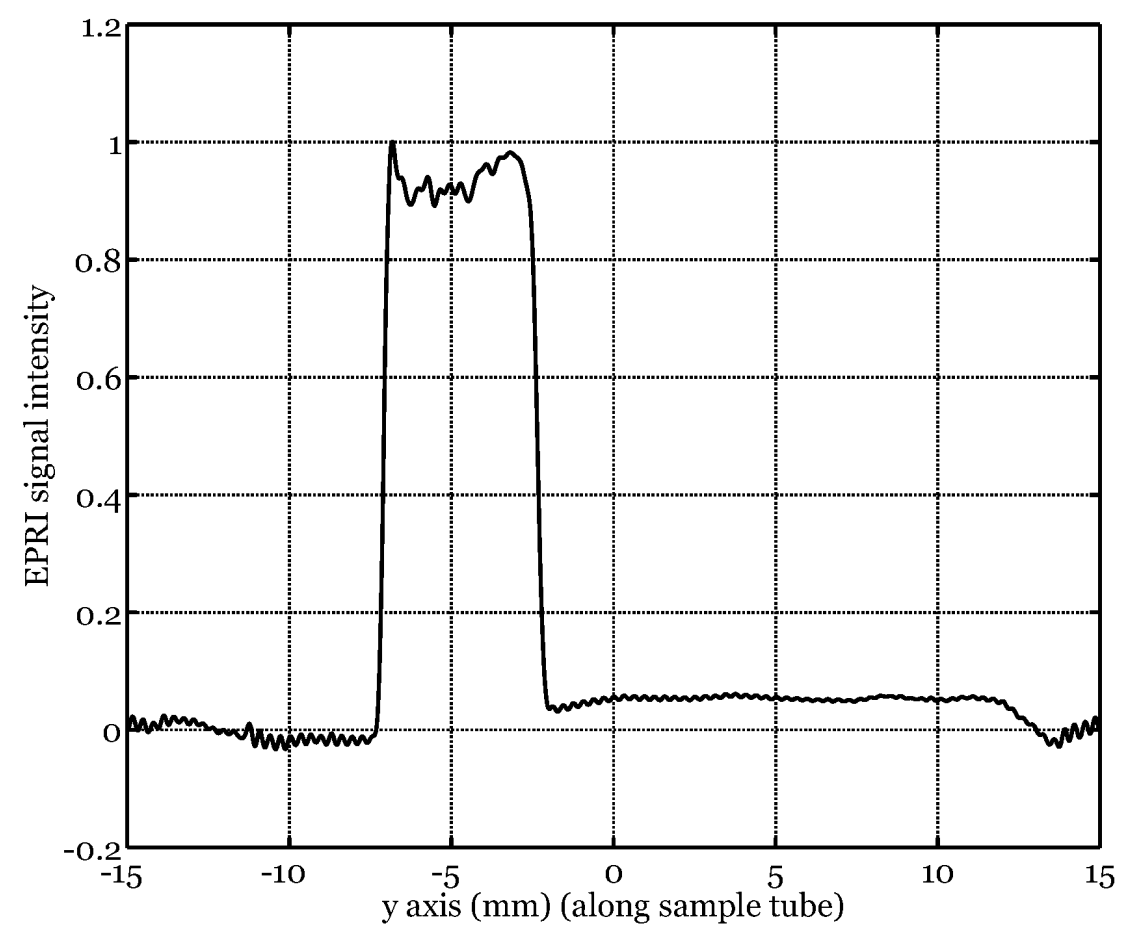

The reconstruction of four $5 \mathrm{~mm}$ homogenously irradiated potassium dithionate tablets is shown in figure $5 \mathrm{~b}$. The spectra used for the deconvolution obtained at zero gradient and at $170 \mathrm{G} / \mathrm{cm}$ respectively are shown in figure $5 \mathrm{a}$. 

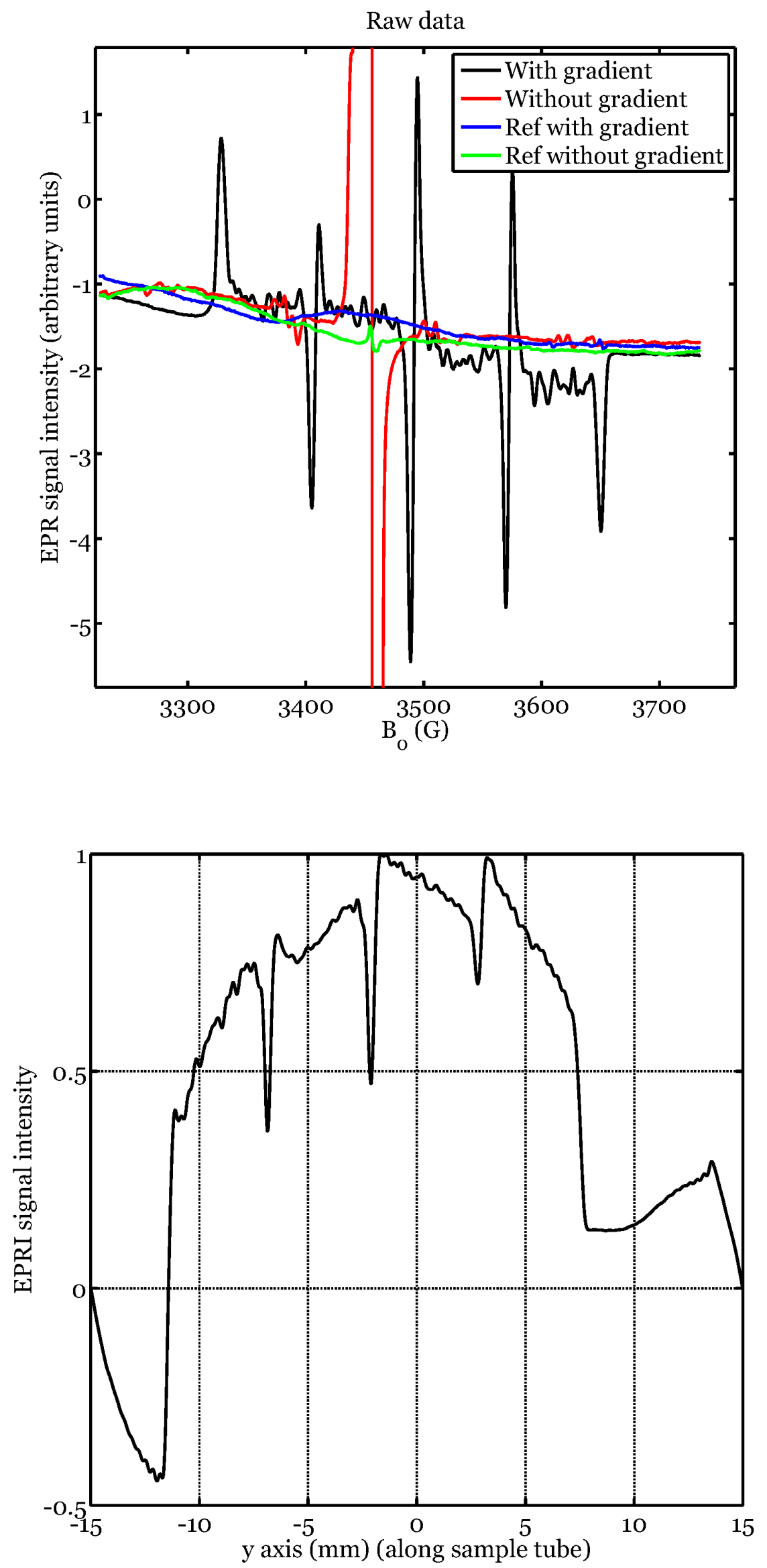
In figure $6 \mathrm{a}$ the left part of the intensity profile from figure $4 \mathrm{~b}$ is magnified showing the slope of the edge for a potassium dithionate tablet after all corrections, in figure $6 \mathrm{~b}$ is shown a magnification of the left part of the corrected intensity profile obtained from an irradiated lithium formate tablet. Inserted, solid, unbroken lines in both figures are the integral of a Gaussian curve with a FWHM corresponding to the resolution for potassiumdithionate; 0.33 $\mathrm{mm}$ and lithiumformate; $0.7 \mathrm{~mm}$ respectively.

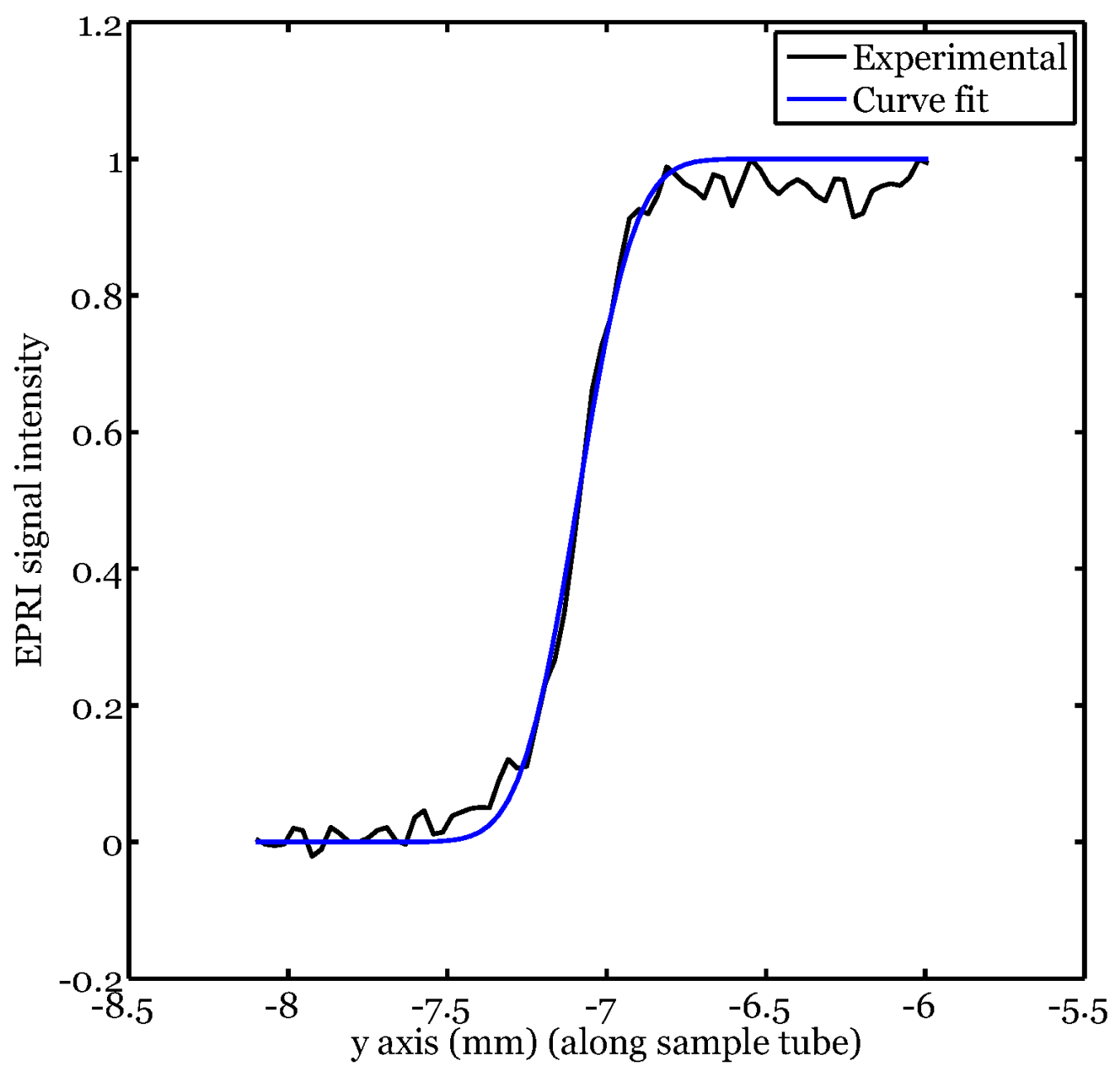




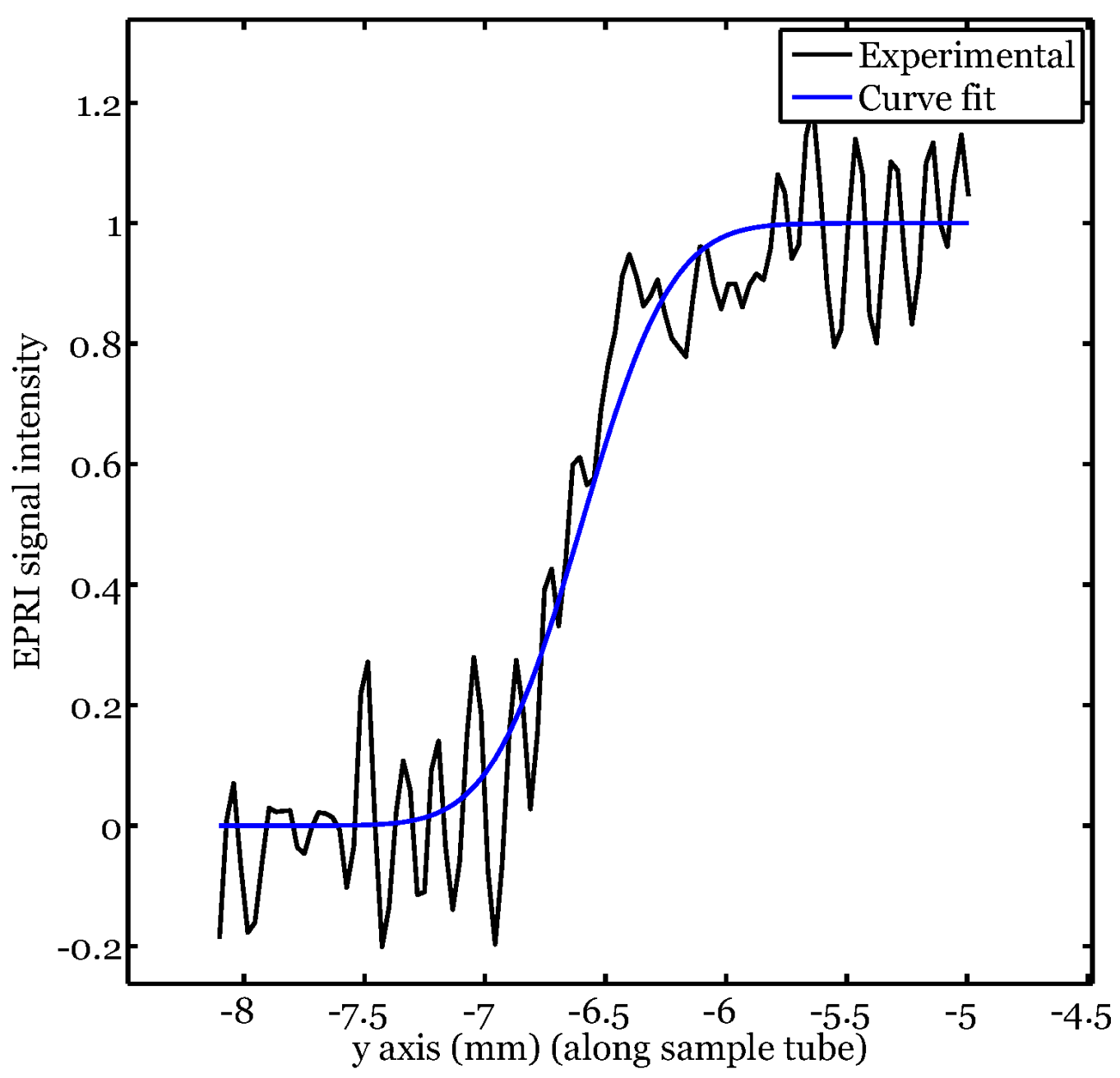

\section{Discussion}

EPR imaging of dose distributions requires a high resolution to be capable of visualization of steep dose gradients. The line width of the EPR spectrum is limiting the resolution (10). Vanea et al.(5) used lithium formate as dosimeter material for imaging of ${ }^{125}$ I seeds embedded in the dosimeter material and found deviations in the dose profiles when compared to Monte Carlo calculations probably due to the low resolution. The line width of lithium formate is $14 \mathrm{G}$ resulting in a resolution of $4.6 \mathrm{~mm}$ with a gradient of $30 \mathrm{G} / \mathrm{cm}$. Kolbun et al.(6) improved the resolution of the EPR imaging by using ammonium formate with narrower spectral lines in a gradient field of $45 \mathrm{G} / \mathrm{cm}$ and got a good agreement of the radial dose values with both Monte Carlo calculations and film dosimetry. Both these studied were performed with 2D imaging using L-band. 
For the present investigation the main purpose was to map dose profiles and 1D EPR imaging was used with high gradient field in X-band. The importance of narrow line widths is verified in the comparison of the edge spread function for potassium dithionate with that for lithium formate fig $6 a$ and $b$.

In the present post processing a Gaussian filtering function corresponding to a point spread function, PSF, with a FWHM of $0.2 \mathrm{~mm}$ in the spatial domain is used. The resulting spatial resolution is $0,33 \mathrm{~mm}$ and no further deconvolution was performed. Anton and Selbach (8) achieved a much higher resolution $0,1 \mathrm{~mm}$ for alanine. They compared the experimental dose distribution with a Monte Carlo simulated one convolved with a Gaussian PSF and found a good correspondence when the FWHM of the PSF was $0.1 \mathrm{~mm}$ and with a signal to noise ratio around 500. In a study of super resolution continuous wave EPR Hirata et al. (15) showed that with use of an iterative deconvolution technique the spatial resolution of a dose profile could be improved up to five fold. This technique is successful when the dose profile could be estimated. For the aim at deciding unknown dose distributions an estimation is more complicated which is the reason why we at present did not include it in the reconstruction procedure.

The main radicals in irradiated potassium dithionate are two $\cdot \mathrm{SO}_{3}^{-}$radicals (16). The corresponding lines in the EPR spectrum are from the superposition of two isotropic signals with slightly different $g$ values. This results in a high yield and a quite narrow line-width which is favorable for EPR imaging as pointed out by Anton and Selbach (8).

However it is not only the line-width that causes degradation of the images but also imperfections in the baselines for the spectra. The quite weak spectrum obtained in the high gradient field is deconvoluted with a much stronger spectrum obtained without gradient and small imperfections will thereby be enhanced. Some imperfections like those shown in figure 3 are outside the interesting part of the spectra but will after the deconvolution cause incorrect slopes in the concentration profiles which can be avoided with careful base line corrections, shown in fig 4 a.

When reconstructing the radical density profile of a homogenously irradiated tablet the spectrum obtained with the gradient field looks like starting with an upper lobe of the EPR spectrum and finish with a lower lobe at the end of the tablet, see figure 2 . This is a summation of an array of spectra for every slice of the tablet but if the area of the upper lobe is the same as the area of the lower lobe they will cancel and between the endpoints we 
expect a straight line. However the spectrum obtained at a gradient of $170 \mathrm{G} / \mathrm{cm}$ (black) in figure 2 shows a rather large noise level probably caused by incomplete compensation between the upper and lower lobes of the spectral information along the irradiated tablet. This effect might partly be explained by the fact that it is the potassium dithionate crystals that contain the radicals and not the space between them. In the deconvolution process this noise level will be pronounced and result in a high variation in the radical density. Anton and Selbach, (8), found that when usisng the Fourier method the noise tends to be amplified by the deconvolution process and that a maximum entropy algorithm appeared to exhibit smaller artifacts. By careful baseline restoration we decreased the noise or the artifacts but not completely and in the future more sophisticated reconstruction algorithms will be used.

Our intentions were to visualize a region of $20 \mathrm{~mm}$ using a stack of four $5 \mathrm{~mm}$ tablets as shown in figure 5. The small gap between the tablets will however be pronounced for the same reason as described above. The inhomogenous sensitivity of the cavity shows a large variation over $20 \mathrm{~mm}$; only about +/-2 $\mathrm{mm}$ around the center of the cavity can be regarded to have a constant sensitivity. At $10 \mathrm{~mm}$ from the center the sensitivity is less than $50 \%$ resulting in too large corrections which enhance the noise and the imperfections in base line corrections. The narrow gaps between the tablets are severely enhanced and large artifacts occur when the difference is big between the area under the upper lobe in the EPR spectrum from one tablet and the lower lobe from the next tablet. This problem can only be avoided if longer homogenous tablets are used instead of a composition of $5 \mathrm{~mm}$ tablets.

\section{Conclusion}

We conclude that the EPR imaging technique needs improvements mainly regarding the reconstructions before being competitive with other methods for mapping steep dose gradients accurately. However within $\pm 5 \mathrm{~mm}$ from the center of the cavity it is possible to visualize a dose distribution with an accuracy of about $5 \%$. With this accuracy dose distributions can be visualized with EPR imaging at X-band using potassium dithionate tablets 5 to $10 \mathrm{~mm}$ height irradiated in stacks, combined with absolute dosimetry performed in selected points with well calibrated tissue equivalent lithium formate dosimeters.

Acknowledgments

This work was supported by grants from the Swedish Cancer foundation (CF) contract number 110322 and The Swedish Research Council (diarienr 2009-5430). We thank prof A 
Lund for skillful synthesizing of potassium dithionate and ass. prof $\AA$ Carlsson Tedgren for performing the irradiations.

\section{References:}

1. Olsson S K, Bergstrand E S, Carlsson Å K, Hole E O and Lund E, Radiation dose measurements with alanine/agarose gel and thin alanine films around a ${ }^{192} \mathrm{Ir}$ brachytherapy source, using ESR spectroscopy, Phys. Med. Biol. 47, 1333-56 (2002).

2. Antonovic L, Gustafsson H, Alm Carlsson G and Carlsson Tedgren Å, Evaluation of a lithium formate EPR dosimetry system for dose measurements around ${ }^{192} \mathrm{Ir}$ brachy therapy sources, Medical Physics, 36(6), 2236-47 (2009).

3. Gustafsson $\mathrm{H}$, Lund E, and Olsson S, Lithium formate EPR dosimetry for verifications of planned dose distributions prior to intensity-modulated radiation therapy. Phys. Med. Biol. 53 4667-82 (2008).

4. Waldeland E, Hörling M, Hole E O, Sagstuen E and Malinen E, Dosimetry of stereotactic radiosurgery using lithim formate EPR dosimeters, Phys. Med. Biol, 55, 2307-16 (2010).

5. Vanea E S, Levêque ., Abboud F, Bol A, Denis J. M, Kolbun N, Vynckier S, and Gallez B. Evaluation of the Dose Distribution Gradient in the Close Vicinity of Brachytherapy Seeds Using Electron Paramagnetic Resonance Imaging. Magnetic Resonance in Medicine. 61 1225-31 (2009).

6. Kolbun N, Levêque P, Abboud F, Bol A, Vynckier S, and Gallez B., Experimental determination of the radial dose distribution in high gradient regions around ${ }^{192} \mathrm{Ir}$ wires: Comparison of electron paramagnetic resonance imaging, films, and Monte Carlo simulations. Med. Phys. 37,5448-55 (2010)

7. Levêque P, Godechal Q, Bol A, Trompier F, and Gallez B, X-band EPR imaging as a tool for gradient dose reconstruction in irradiated bones. Med. Phys. 36 pp 4223-29 (2009).

8. Anton M and Selbach H-J, Measurements of dose distributions in alanine using EPR imaging. Bruker Spin Report 157-158, 48-51 (2006)

9. Gustafsson H, Kruczala K, Lund E and Schlick S, Visualization of Dose Distribution and Linear Energy Transfer by 1D and 2D Spectral-Spatial ESR Imaging: A Potassium Dithionate Dosimeter Irradiated with $\mathrm{C}^{6+}$ and $\mathrm{N}^{7+}$ Ions. The Journal of Physical Chemistry B 112, 8437-42 (2008)

10. Karthe W and Wehrsdorfer E, The Measurement of Inhomogeneous Distributions of Paramagnetic Centers by Means of EPR. Journal of Magnetic Resonance 33, 107-11 (1979) 
11. Ohno K, Method of EPR Imaging: Application to Spatial Distributions of Hydrogen Atoms Trapped in Sulfuric Acid Ices. Japanese Journal of Applied Physics. vol. 20, No. 3, pp. L179-L182 1981

12. Ahn K-H, Halpern H J, Simulation of 4D spectral-spatial EPR images. J Magn Reson 187, 1-9 (2007).

13. Stoll S and Schweiger A, EasySpin, a comprehensive software package for spectral simulation and analysis in EPR, Journal of Magnetic Resonance 178, $42-55$ (2006).

14. Höfer P. and Carl P. Method for determining the absolute number of electron spins in a sample of extended size. United States Patent. Patent No.: US 7,586,305 B2, (2009).

15. Hirata H, Wakana M, and Susaki H, Feasibility study of superresolution cintinous-wave electron paramagnetic resonance imaging, Applied Physics Letters 88, 254103,(2006)

16. Gustafsson H, Lund A, Hole E O and Sagstuen E, SO3-radicals for EPR dosimetry; X- and Q band EPR study and LET dependencey of crystalline potassium dithionate, Radiation Measurements, 2013, 59, 123-128. 